\title{
Diversas estrategias resultarian efectivas para controlar problemas del comportamiento nocturno en niños pequeños
}

Ramchandani P, Wiggs L, Webb V et al. A Systematic Review of Treatments for Settling Problems and Night Waking in Young Children. BMJ 2000;320:209-13

\section{Objetivo}

Comparar la eficacia de diversas estrategias para disminuir el llanto nocturno y optimizar la puesta a dormir en niños pequeños.

\section{Fuente de Datos}

Dos de los autores analizaron en forma independiente estudios clínicos de varias bases de datos electrónicas (MEDLINE, Embase y Cochrane.) utilizando los términos: niño, infante, lactante, dormir, tratamiento, medicación y despertar. Luego se amplió la búsqueda hacia las referencias citadas en estos trabajos y en trabajos de revisión y en libros de texto pertinentes. Para detectar estudios no publicados fueron entrevistados los autores de todos los estudios hallados y el fabricante de la trimeprazina (un antihistamínico).

\section{Selección de Estudios}

Se seleccionaron estudios controlados y aleatorizados de niños de hasta 5 años con problemas de comportamiento nocturno, ya sea en la puesta a dormir (rechazo, berrinches, tardanza excesiva) o durante la noche (llanto nocturno, despertares frecuentes o prolongados durante la madrugada). La calidad metodológica de los estudios fue evaluada en forma independiente por dos de los autores utilizando el sistema de Jadad que otorga puntajes del 1 al 5.

\section{Extracción de Datos}

Se seleccionaron 9 estudios que cumplieron con los criterios de inclusión y cuya rigurosidad metodológica era aceptable ( 4 con fármacos y 5 sobre tratamientos comportamentales o educativos). El puntaje promedio de la calidad de los estudios fue bajo (2,1 puntos). Fue imposible efectuar la valoración de los datos en forma de meta análisis dada la heterogeneidad de los sujetos, los tratamientos y los resultados principales medidos en cada estudio.

Fuente de financiamiento: Diversos organismos gubernamentales.

\section{Resultados Principales}

Estrategias Farmacológicas: Dos estudios que compararon la trimeprazina versus placebo demostraron una reducción estadísticamente significativa (aunque clínicamente menos clara) en el número de despertares nocturnos en el corto $p$ lazo ( $p<0,01$ y $p<0,001$ respectivamente).

Un tercer estudio que comparó la mioprazina (un antihistamínico) contra el clordesmetil diacepam demostró que ambos son igualmente efectivos para reducir la frecuencia de despertares nocturnos ( 88 al $100 \%$ de efectividad). Elúltimo trabajo comparó durante 4 semanas el método de extinción más trimeprazina versus el método de extinción más placebo. Los niños del grupo intervención redujeron sus despertares mas rápidamente durante los primeros días sin encontrarse diferencias hacia el final del estudio. Estrategias no Farmacológicas (ver abajo): Las técnicas de despertares programados y la extinción redujeron de igual forma respecto al grupo control los despertares nocturnos $(p<0,05)$.

Comparadas con los controles, la utilización de rutinas positivas y de extinción graduada demostraron ser igualmente efectivas en la reducción del número y duración de los berrinches a la hora de dormir $(p<0,001)$.

El único trabajo que no contó con grupo control demostró que la extinción modificada fue útil para disminuir el número de despertares nocturnos. El programa individualizado demostró ser efectivo en disminuir el llanto nocturno $(p<0,05)$, no así la entrega de un folleto educativo no directivo.

\section{Conclusiones}

A pesar de que el tratamiento farmacológico (en especial con trimeprazina) parecería ser una medida efectiva en el corto plazo, las intervenciones no farmacológicas se presentan como más efectivas tanto en el corto como el mediano plazo.

A pesar de que la extinción sería mas rápidamente efectiva, ninguna de las técnicas comportamentales ha demostrado ser superior en el mediano plazo (luego de al menos 6 semanas de seguimiento)

Las conclusiones resultantes deben considerarse con precaución dada la pobreza metodológica y el $n$ reducido de los estudios analizados en ella..

\section{COMENTARIO}

Se estima que al menos el 44\% de los lactantes de 9 meses presentan despertares nocturnos (definido como aquel episodio de despertar que requiere de la intervención del cuidador para serenarlo). En un 25\% de los casos estos resultan frecuentes ( 7 o más por semana) y potencialmente problemáticos para los padres ${ }^{1}$. Junto con los problemas a la hora de dormir constituyen objeto de preocupación y de habitual consulta en el ámbito de la atención primaria pediátrica. Habiéndose establecido que la intervención activa de los padres en dormir al lactante desencadena mecanismos condicionantes que imposibilita a este dormirse por sus propios medios es que la técnica de la extinción era la intervención que contaba con mayor fuerza de evidencia ${ }^{2-3}$. Esta revisión presenta otras estrategias igualmente efectivas.

La técnica de extinción graduada y de rutinas positivas surgen como las únicas alternativas para controlar los problemas surgidos a la hora de dormir.

Las intervenciones farmacológicas demostraron ser útiles únicamente en el corto plazo y fueron realizadas con antihistaminicos y una benzodiazepina que no se disponen en el mercado Local; quedarían reservadas para aquellas situaciones en que los padres no acepten las técnicas comportamentales.

Las Intervenciones No Farmacológicas son:

Técnicas para controlar la puesta a dormir

-Rutinas Positivas: 20 minutos antes de hora en que habitualmente el niño se duerme se pone en marcha una rutina de apaciguamiento que se adelanta $5 \mathrm{a}$

10 minutos por semana hasta llegar a un horario apropiado.

-Extinción Graduada: se utiliza en aquellos niños que reaccionan con berrinches a la hora de dormir. Consiste en ignorarlos durante un número predeterminado de minutos, que se van prolongado semana a semana.

Técnicas para controlar los despertares nocturnos

- Despertares programados: luego de obtener un registro basal del patrón de sueño nocturno del lactante los padres deben despertarlo 15 a 60 minutos antes del horario en que este lo haría espontáneamente.

-Extinción: se instruye a los padres que ante cada episodio de llanto nocturno acudan ante el niño, verificando su bienestar pero sin interactuar con él de ninguna forma.

-Extinción Modificada: similar a la técnica anterior, pero los padres acuden ante el niño luego de 20 minutos de llanto. El procedimiento se repite cada 20 minutos hasta que este duerma nuevamente.

-Folleto Educativo: se entrega a los padres material impreso con información general, no directiva sobre los problemas del comportamiento nocturno en los niños y las ventajas y desventajas de diversas estrategias para su control. -Programa Individualizado: consistente en adecuar al contexto particular de cada familia un programa utilizando una o varias de las estrategias no farmacológicas complementadas con la entrega de material impreso y con llamados telefónicos de apoyo.

\section{Dr. Sebastián Fernández}

Residente de Medicina Familiar

Unidad de Medicina Familiar y Preventiva. Hospital Italiano de Buenos Aires

\section{Referencias}

1. Nightwaking in infants during the first year of life. Anders TF. Pediatrics 1979;63:860-64

2. Nightwaking during infancy: the role of parental presence at bedtime. Adair R, Bauchner H, Levenson S, et al. Pediatrics 1991;87:500-04

3. Well-Baby care in the first 2 years of life. Feldman W. Canadian Task Force on the Periodic Health Examination. Canadian Guide to Clinical Preventive Health Care. 0 ttawa: Health Canada, 1994;258-66 Palabras Claves

- problemas del comportamiento nocturno - llanto nocturno - despertares frecuentes - extinción 